\title{
Pupillary Response to Auditory Stimuli in Depressive State
}

\author{
Hiroaki Oguro $^{\text {a, d }}$, Nobuo Suyama ${ }^{\mathrm{b}}$, Kenji Karino ${ }^{\mathrm{c}}$, Shuhei Yamaguchi ${ }^{\mathrm{a}}$
}

\begin{abstract}
Background: Previous reports indicate that depressed individuals have worse memory for negative information than other types of information. They tend to ruminate upon negative information with high sensitivity. Depressive subjects also tend to display greater sustained pupil dilation in response to negative information.

Methods: We evaluated pupil diameter with charge coupled device (CCD) infrared camera while scoring depressive scale in 41 healthy elderly subjects. Six kinds of sounds were used as auditory stimuli with characters of startle-eliciting. The measurements of pupil were made in a rested state and done twice with pre- and post-auditory and light stimulations. The indexes of pupil responses were dilations for auditory stimulations and contractions for light stimulations.
\end{abstract}

Results: The pupil diameter stimulated by emotional sound "glasses broken on the floor" was solely positive correlated with depressive score. The pupil contractions for light stimuli were weakly negative correlated with depressive score.

Conclusion: Some emotional sounds with negative information could cause pupil dilation in depressive participants. This sound "glasses broken on the floor" would be a biological marker assistant to depressive diagnosis.

Keywords: Depression; Pupil; Dilation; Contraction; Pupillary response; CCD camera; Auditory stimuli; Negative information; Zung's self-depression scale

\section{Introduction}

Pupillary diameter is controlled by the combined activity of the sympathetic and parasympathetic nervous system with the cen-

Manuscript accepted for publication January 21, 2016

${ }^{a}$ Department of Neurology, Faculty of Medicine, Shimane University, Japan ${ }^{b}$ Tsuwano Kyozon Hospital, Kanoashi-gun, Shimane, Japan

${ }^{\mathrm{c} C l i n i c a l}$ Skill Up Center, Faculty of Medicine, Shimane University, Japan ${ }^{\mathrm{d} C}$ Corresponding Author: Hiroaki Oguro, Department of Neurology, Faculty of Medicine, Shimane University, 89-1 Enya-cho, Izumo 693-8501, Japan. Email: oguro@med.shimane-u.ac.jp

doi: http://dx.doi.org/10.14740/jnr364w tral nervous systems [1-3]. Pupil dilations may generate in response to changes in light, emotional auditory stimuli, convergence-accommodation, sensory inputs from the surface body and many tasks $[4,5]$. In animal experiments, stimulation of the frontal cortex and the sensorimotor area led to pupillary dilation [4]. Otherwise behavioral and psychological syndromes were essential problems to diagnose dementia at an early stage in elderly peoples [6]. Many studies have reported that pupil dilation was correlated with cognitive load under conditions of higher attentional allocation, memory use, interpretation of difficult material and mental arithmetic [7]. Siegle et al reported depressive individuals tended to display sustained pupil dilation in response to negative stimuli [8]. It has been gradually elucidated whether pupil response would detect mood disorder, especially depression or not $[9,10]$. We had previously reported the dilation of the pupils induced from various emotional auditory stimuli related to the cortical and subcortical mechanisms [11]. In this study, we evaluated pupil response to auditory and directive light stimuli in 41 healthy elderly people. And we analyzed pupillary responses compared with depression.

\section{Subjects and Methods}

Subjects were 41 healthy elderly people (mean age $74.3 \pm 6.7$ years) who had no medication with chronic disease and had no disorder and operative history in pupil and iris. They also had no history of alcohol or substance abuse, and no current use of psychoactive medications. The size of pupil was monitored by Iriscorder ${ }^{\circledR}$ C7364 (Hamamatsu Photonics, Hamamatsu, Japan) with a charge coupled device (CCD) infrared camera. Binocular infrared video pupillography was used to non-invasively measure the sympathetic and parasympathetic nerves in the pupils. Light emitting diode was lit in the goggle. Data analysis was studied with MesEye Version 1.32 installed to Windows PC note (SONY PCG-FR77). Depressive degree was evaluated with Zung's self-depression scale (SDS) [12].

The sounds used as auditory stimuli had characters of several kinds of startle-eliciting and its intensity was $70 \mathrm{~dB}$ above individual threshold. We adopted six sound series: "free fall", "glasses broken on the floor", "introduction of music", "gunshot", "car passing" and "scream". The light stimulus was 660 $\mathrm{nm}$ and $10 \pm 3 \mu \mathrm{W}$ with $1 \mathrm{~s}$. After dark adaptation of $15 \mathrm{~min}$, the subjects were installed with monitoring camera. The measurements of pupil were made in a rested state and done twice, pre- and post-stimulations. We examined all subjects with right pupil. 


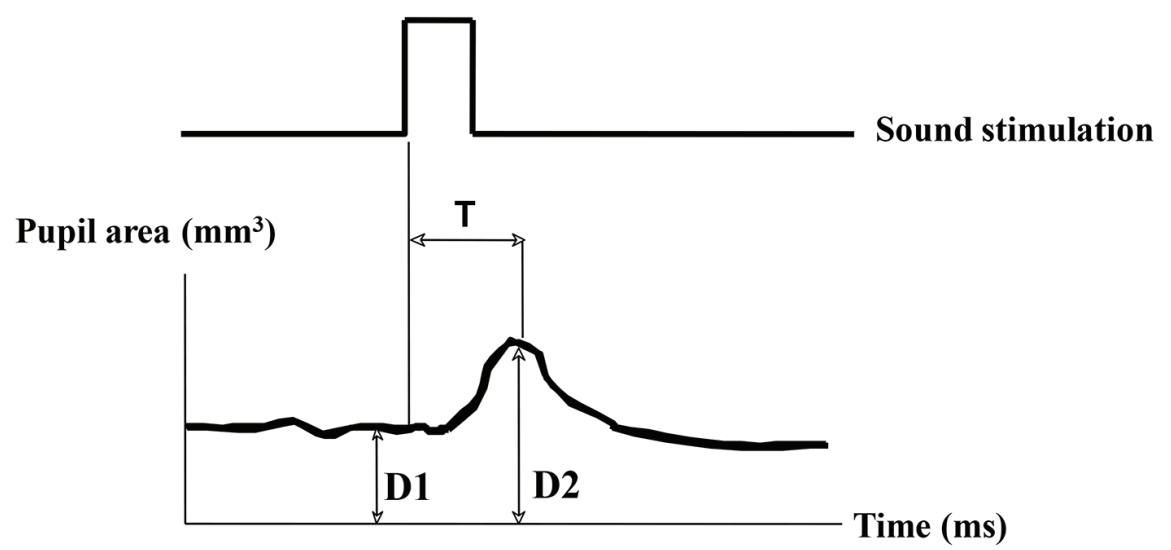

Figure 1. Measurement of pupil responsive area after sound stimulation. D1 $\left(\mathrm{mm}^{2}\right)$ : pupil area of pre-stimulant with mean value for $1 \mathrm{~s}$. D2 ( $\left.\mathrm{mm}^{2}\right)$ : pupil area of post-auditory stimulant with maximum value. Dilated ratio (DR) is (D2 - D1)/D1. T is a latency time between sound stimulation and post-auditory response with maximum (ms).

The indexes of pupil responses were defined as follows. D1 $\left(\mathrm{mm}^{2}\right)$ was initial area of pre-stimulation with mean value for $1 \mathrm{~s}$ preceding the onset of the stimulus. D2 $\left(\mathrm{mm}^{2}\right)$ was maximum area of post-auditory stimulation. Dilated ratio $(\mathrm{DR})$ was $(\mathrm{D} 2-\mathrm{D} 1) / \mathrm{D} 1 \times 100$. T was a latency time between sound stimulation and post-auditory and maximum response with maximum (ms) (Fig. 1). D3 $\left(\mathrm{mm}^{2}\right)$ was defined as pupil contraction with minimum area of post-light stimulation. Contractive ratio (CR) was (D1 - D3)/D1 (Fig. 2). Statistical analysis was used with Pearson correlation coefficient and less than 0.05 was estimated statistically significant.

\section{Results}

The mean score of SDS was $29.6 \pm 5.6$ (minimum score was 20 and maximum score was 45 ). The clinically depressive subjects who had more than 40 score in SDS are only three
(7.3\%). The DR in broken glasses sound was statistically positive correlated with SDS $(\mathrm{r}=0.314, \mathrm{P}=0.0422)$ (Fig. 3A). The CR of light stimuli was weakly negative correlated with SDS ( $r=-0.294, P=0.0651)$ (Fig. 3B). The DRs in other five sounds had no correlation with SDS. The latencies of pupillary responses were not significant among kinds of sound series. Average latency of dilated response was $0.3 \mathrm{~s}$. T was not significant among all stimulations.

\section{Discussion}

Pupil dilation increased cognitive load such as auditory task. Pupil responses are classified two phases according to duration [2]. Antikainen and Niemi reported that subjects in neuroticism displayed larger pupil dilation under loud noise because of individual differences [13]. Karatekin et al reported that task-evoked pupillary responses (TEPRs) studies would
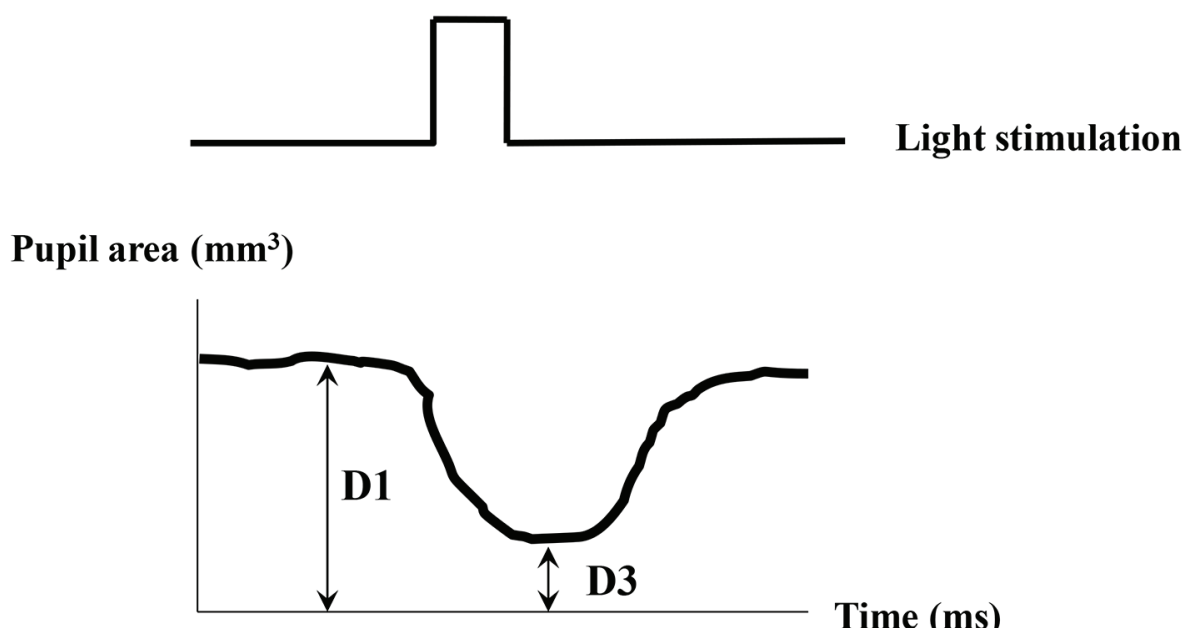

\section{Time (ms)}

Figure 2. Measurement of pupil responsive area after light stimulation. D1 $\left(\mathrm{mm}^{2}\right)$ : pupil area of pre-stimulant with mean value for $1 \mathrm{~s} . \mathrm{D} 3\left(\mathrm{~mm}^{2}\right)$ : pupil minimum area of post-light stimulation. Contractive ratio (CR) is (D1 - D3)/D1. 
A

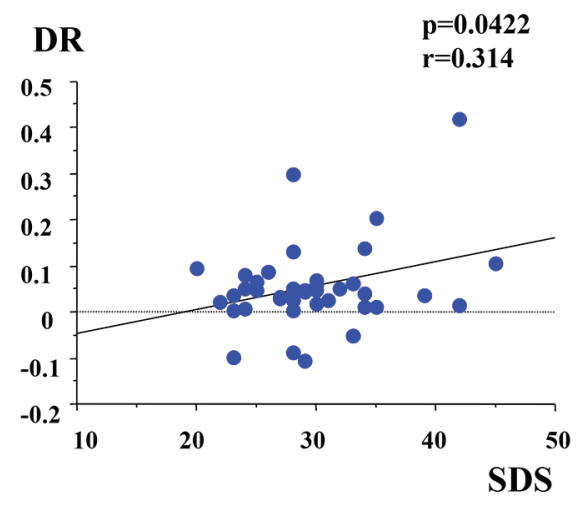

B

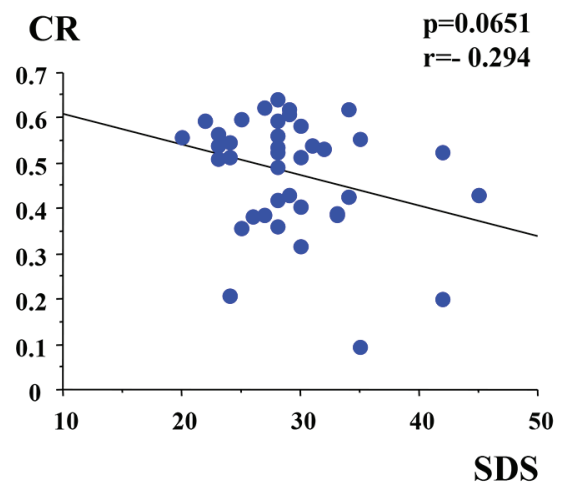

Figure 3. (A) Correlation between DR and SDS. DR: dilated ratio; SDS: Zung's self-depression scale. (B) Correlation between CR and SDS. CR: contractive ratio.

be suitable for investigating the effects of arousal (e.g., from fatigue, drowsiness, stress, caffeine, or medications) on allocation of attention. They also suggested that tonic changes in pupillary diameter were influenced by general factors, such as level of emotional arousal, anxiety and stress. Increased anxiety and fatigue might reflect in elevated catecholamine and cortisol levels secondary to the pupil responses [1]. Pupil responses would be caused by emotional sounds, so we hypothesized that pupil dilation might be clinical indexes that enable us to inform diagnosis mood disorder.

Depressive individuals' pupillary dilations are obtained on multiple well-known emotional information. Why did their pupils only respond to the sound of "glasses broken on the floor" in our study? Siegle et al employed emotional processing tasks (lexical decision and valence identification) as s target stimulus materials to correlate pupil dilation with depression, and indicated that depressive subjects tend to display greater sustained pupil dilation in response to tasks requiring participants to attend to the emotional content of words. They also explained that depressed individuals have worsen memory for negative information than other types of information, so their increased attention to negative information appears as rumination, particularly depressive symptoms [8]. Glasses broken sound might be one of negative information and tend to make subjects to think negatively.

Direct cortical and indirect cortico-thalamic-hypo-thalamic pathways producing inhibition at the Edinger-Westphal region cause relaxation of the sphincter muscles and papillary dilation [14]. Limbic lesions, such as amygdala also output parasympathetic pathways inhibiting of pupillary constrictor muscles, so stimulation of limbic regions increases pupil dilation [10]. Niehaus et al reported that cortex stimulation by repetitive transcranial magnetic stimulation (rTMS) causes pupil dilation due to inhibition of the parasympathetic and activation of the central sympathetic system [4]. They supposed that stimulation over the right central region evoking larger dilation of the pupil, which might be one of the cortical pupillmotor centers, but finally concluded papillary responses to rTMS are only unspecific activation of the sympathetic system. Depressed individuals decreased prefrontal activity, particularly in the dorsolateral prefrontal region and dopamine availability decreases in the prefrontal cortex [15]. Otherwise Jorge et al reported that rTMS of the left dorsolateral prefrontal cortex reduces depressive symptoms in 20 post-stroke depression [16]. Frontal cortex and limbic lesions could be an important center linking pupil response with depressive state.

Our results showing depressed subjects tend to increase the constrictive pupil area are greater than not depressed. It is consistent with a previous report that the pupil area at the peak of constriction is greater in the depressed subjects compared with the normal controls due to decreasing of acetyl cholinergic activity in depression [9]. Other report denies that the difference of acetyl cholinergic activity between depression and normal controls [17]. The pupillary light response is complex, because noradrenaline activity also works on controlling the response. In addition to depression, aging causes the loss of cortical noradrenergic neurons and lower level of noradrenaline activity. Existence of lower level of noradrenaline and acetylcholine activity in depressed subjects affects in pupillary light reflex [9].

Our experimental subjects have not so much depressive condition, so we could have only referred to potentially and pre-depressive state. The pupil diameter is likely to reflect an increase in sympathetic activity, so it would be better for relaxation of participants' mentality. We might have some problems in experimental setting to be ameliorated ahead of the auditory stimuli which autonomic condition needs stable as much as possible. For example, we had better to let the participants to hear comfortable sounds such like song of a canary and murmur of a stream, so they can keep them to be relax with domination in parasympathetic nerve system.

This is, to our knowledge, the first study on this point that correlation with pupil response and depression is estimated with auditory stimuli with negative information.

\section{Conclusion}

We suggest that some startle-eliciting sound might cause pupillary dilated response in depressive person. It may be a bio- 
logical marker assistant to depressive diagnosis.

\section{Conflict of Interest}

The authors have no conflict interest.

\section{References}

1. Karatekin C, Couperus JW, Marcus DJ. Attention allocation in the dual-task paradigm as measured through behavioral and psychophysiological responses. Psychophysiology. 2004;41(2):175-185.

2. Hirano T, Inoue H, Uemura T, Matsunaga K. Pupillary responses in normal subjects following auditory stimulation. Eur Arch Otorhinolaryngol. 1994;251(Suppl 1):S36.

3. Lowenstein O, Loewenfeld IE. Role of sympathetic and parasympathetic systems in reflex dilation of the pupil; pupillographic studies. Arch Neurol Psychiatry. 1950;64(3):313-340.

4. Niehaus L, Guldin B, Meyer B. Influence of transcranial magnetic stimulation on pupil size. J Neurol Sci. 2001;182(2):123-128.

5. Berthold HC, Slowiaczek ML. Visual significance of pupillary response to an auditory task. Percept Mot Skills. 1975;41(3):821-822.

6. Mirakhur A, Craig D, Hart DJ, McLlroy SP, Passmore AP. Behavioural and psychological syndromes in Alzheimer's disease. Int J Geriatr Psychiatry. 2004;19(11):1035-1039.

7. Granholm E, Asarnow RF, Sarkin AJ, Dykes KL. Pupillary responses index cognitive resource limitations. Psychophysiology. 1996;33(4):457-461.

8. Siegle GJ, Granholm E, Ingram RE, Matt GE. Pupil- lary and reaction time measures of sustained processing of negative information in depression. Biol Psychiatry. 2001;49(7):624-636.

9. Wang J, Fan Y, Zhao X, Chen N. Pupillometry in Chinese female patients with depression: a pilot study. Int J Environ Res Public Health. 2014;11(2):2236-2243.

10. Silk JS, Dahl RE, Ryan ND, Forbes EE, Axelson DA, Birmaher B, Siegle GJ. Pupillary reactivity to emotional information in child and adolescent depression: links to clinical and ecological measures. Am J Psychiatry. 2007;164(12):1873-1880.

11. Suyama N, Kobayashi S, Yamaguchi S, Okada K, Nitta M. Pupillary response to auditory stimuli. Neuro-ophthalmol Jpn. 1998;15:305-310.

12. Zung WW. A Self-Rating Depression Scale. Arch Gen Psychiatry. 1965;12:63-70.

13. Antikainen J, Niemi P. Neuroticism and the pupillary response to a brief exposure to noise. Biol Psychol. 1983;17(2-3):131-135.

14. Steinhauer SR, Siegle GJ, Condray R, Pless M. Sympathetic and parasympathetic innervation of pupillary dilation during sustained processing. Int J Psychophysiol. 2004;52(1):77-86.

15. Baxter LR, Jr., Schwartz JM, Phelps ME, Mazziotta JC, Guze BH, Selin CE, Gerner RH, et al. Reduction of prefrontal cortex glucose metabolism common to three types of depression. Arch Gen Psychiatry. 1989;46(3):243-250.

16. Jorge RE, Robinson RG, Tateno A, Narushima K, Acion L, Moser D, Arndt S, et al. Repetitive transcranial magnetic stimulation as treatment of poststroke depression: a preliminary study. Biol Psychiatry. 2004;55(4):398-405.

17. Fountoulakis K, Fotiou F, Iacovides A, Tsiptsios J, Goulas A, Tsolaki M, Ierodiakonou C. Changes in pupil reaction to light in melancholic patients. Int J Psychophysiol. 1999;31(2):121-128. 